T. Hiramatsu and S. Akiyama

Nagoya Math. J.

Vol. 111 (1988), 157-163

\title{
ON SOME DIMENSION FORMULA FOR AUTOMORPHIC FORMS OF WEIGHT ONE III
}

\author{
TOYOKAZU HIRAMATSU AND SHIGEKI AKIYAMA \\ Dedicated to Professor Michio Kuga on his 60th birthday
}

Let $\Gamma$ be a fuchsian group of the first kind and assume that $\Gamma$ does not contain the element $\left(\begin{array}{rr}-1 & 0 \\ 0 & -1\end{array}\right)$. Let $S_{1}(\Gamma)$ be the linear space of cusp forms of weight 1 on the group $\Gamma$ and denote by $d_{1}$ the dimension of the space $S_{1}(\Gamma)$. When the group $\Gamma$ has a compact fundamental domain, we have obtained the following (Hiramatsu [3]):

$$
d_{1}=\frac{1}{2} \operatorname{Res}_{s=0} \zeta^{*}(s),
$$

where $\zeta^{*}(s)$ denotes the Selberg type zeta function defined by

$$
\zeta^{*}(s)=\sum_{\alpha=1}^{\infty} \sum_{k=1}^{\infty} \frac{\left(\operatorname{sgn} \lambda_{0, \alpha}\right)^{k} \log \left|\lambda_{0, \alpha}\right|}{\left|\lambda_{0, \alpha}^{k}-\lambda_{0, \alpha}^{-k}\right|}\left|\lambda_{0, \alpha}^{k}+\lambda_{0, \alpha}^{-k}\right|^{-s} .
$$

Here, $\lambda_{0, \alpha}$ denotes the eigenvalue $\left(\left|\lambda_{0, \alpha}\right|>1\right)$ of representative $P_{\alpha}$ of the primitive hyperbolic conjugacy classes $\left\{P_{\alpha}\right\}$ in $\Gamma$.

In this paper we give a formula of the dimension $d_{1}$ for a general discontinuous group $\Gamma$ of finite type such that $\Gamma \nexists\left(\begin{array}{rr}-1 & 0 \\ 0 & -1\end{array}\right)$, by using the Selberg trace formula (Selberg [5], Kubota [4]). ${ }^{1}$

The notation used here will generally be those of [1].

\section{$\S 1$. The Selberg eigenspace $\mathscr{M}\left(1,-\frac{3}{2}\right)$, Eisenstein series and continuous spectrum}

1.1. Let $\Gamma$ be a fuchsian group of the first kind not containing the element $\left(\begin{array}{rr}-1 & 0 \\ 0 & -1\end{array}\right)$, and suppose that $\Gamma$ has a non-compact fundamental

Received February 9, 1987.

1) For the dimension $d_{1}$ in the case of $\Gamma_{\ni}\left(\begin{array}{rr}-1 & 0 \\ 0 & -1\end{array}\right)$, refer to Hiramatsu [2]. 
domain in the upper half plane $S$. Let $T$ be the real torus and put $\tilde{S}=$ $S \times T$. Let $L^{2}(\Gamma \backslash \tilde{S})$ be the space of functions $f(z, \phi)$ on $\tilde{S}$ satisfying the conditions:

1) $f(z, \phi)$ is a measurable function on $\tilde{S}$;

2) $f(g(z, \phi))=f(z, \phi)$ for $g \in \Gamma$;

3) $\int_{\Gamma \backslash \hat{S}}|f(z, \phi)|^{2} d(z, \phi)<\infty$.

Moreover we denote by $\mathscr{M}_{\Gamma}(k, \lambda)=\mathscr{M}(k, \lambda)$ the set of functions $f(z, \phi)$ satisfying the following conditions:

(i) $f(z, \phi) \in L^{2}(\Gamma \backslash \tilde{S})$;

(ii) $\tilde{\Delta} f(z, \phi)=\lambda f(z, \phi),(\partial / \partial \phi) f(z, \phi)=-\sqrt{-1} k f(z, \phi)$,

where

$$
\tilde{\Delta}=y^{2}\left(\frac{\partial^{2}}{\partial x^{2}}+\frac{\partial^{2}}{\partial y^{2}}\right)+\frac{5}{4} \frac{\partial^{2}}{\partial \phi^{2}}+y \frac{\partial}{\partial \phi} \frac{\partial}{\partial x} .
$$

Then the following equality holds (Hiramatsu [2]):

ThEOREM 1. $\mathscr{M}\left(1,-\frac{3}{2}\right)=\left\{e^{-\sqrt{-1} \phi} y^{1 / 2} F(z): F(z) \in S_{1}(\Gamma)\right\} ;$ and hence

$$
d_{1}=\operatorname{dim} S_{1}(\Gamma)=\operatorname{dim} \mathscr{M}\left(1,-\frac{3}{2}\right) .
$$

1.2. We consider an invariant integral operator on the Selberg eigenspace $\mathscr{M}(k, \lambda)$ defined by a point-pair invariant kernel

$$
\omega_{\delta}\left(z, \phi ; z^{\prime}, \phi^{\prime}\right)=\left|\frac{\left(y y^{\prime}\right)^{1 / 2}}{\left(z-\bar{z}^{\prime}\right) / 2 \sqrt{-1}}\right|^{\delta} \frac{\left(y y^{\prime}\right)^{1 / 2}}{\left(z-\bar{z}^{\prime}\right) / 2 \sqrt{-1}} e^{-\sqrt{-1}\left(\phi-\phi^{\prime}\right)}, \quad(\delta>1) .
$$

Then, the integral operator $\omega_{\delta}$ vanishes on $\mathscr{M}(k, \lambda)$ for all $k \neq 1$. It is easy to see that the integral

$$
\int_{\tilde{D}} \sum_{M \in \Gamma} \omega_{\hat{o}}(z, \phi ; M(z, \phi)) d(z, \phi) \quad(\tilde{D}=\Gamma \backslash \tilde{S})
$$

is uniformly bounded at a neighborhood of each irregular cusp of $\Gamma$. We also see that by the Riemann-Roch theorem, the number of regular cusps of $\Gamma$ is even. In the following we assume that $\kappa_{1}, \kappa_{2}$ is a maximal set of regular cusps of $\Gamma$ which are not equivalent with respect to $\Gamma$. Let $\Gamma_{i}$ be the stabilizer in $\Gamma$ of $\kappa_{i}$, and fix an element $\sigma_{i} \in S L(2, R)$ such that $\sigma_{i} \infty$ $=\kappa_{i}$ and such that $\sigma_{i}^{-1} \Gamma_{i} \sigma_{i}$ is equal to the group $\Gamma_{0}=\left\{\left(\begin{array}{cc}1 & m \\ 0 & 1\end{array}\right): m \in Z\right\}$. Then the Eisenstein series attached to the regular cusp $\kappa_{i}$ is defined by 
(2)

$$
E_{i}(z, \phi ; s)=\sum_{\substack{\sigma \in \sum_{i}^{i} \backslash \Gamma \\
\sigma \bar{i}^{2} \sigma=\left(\begin{array}{l}
* \\
c
\end{array}\right)}} \frac{y^{s}}{|c z+d|^{2 s}} e^{-\sqrt{-1}(\phi+\arg (c z+d))} \quad(i=1,2),
$$

where $s=t+\sqrt{-1} r$ with $t>1$. It is easy to check that

(i) $E_{i}(M(z, \phi) ; s)=E_{i}(z, \phi ; s)$ for $M \in \Gamma$;

(ii) $\tilde{\Delta} E_{i}(z, \phi ; s)=\left\{s(s-1)-\frac{5}{4}\right\} E_{i}(z, \phi ; s)$;

(iii) $(\partial / \partial \phi) E_{i}(z, \phi ; s)=-\sqrt{-1} E_{i}(z, \phi ; s)$.

By the above (i), $E_{i}(z, \phi ; s)$ has the Fourier expansion at $\kappa_{j}$ in the form

$$
E_{i}\left(\sigma_{j}(z, \phi) ; s\right)=\sum_{m=-\infty}^{\infty} a_{i j, m}(y, \phi ; s) e^{2 \pi \sqrt{-1} m x} .
$$

The constant term $a_{i j, 0}(y, \phi ; s)$ is given by

$$
\begin{aligned}
e^{\sqrt{-1} \phi} a_{i j, 0}(y, \phi ; s) & =a_{i j, 0}(y ; s) \\
& =\delta_{i j} y^{s}-\sqrt{-1} \sqrt{\pi} \frac{\Gamma(s)}{\Gamma\left(s+\frac{1}{2}\right)} \varphi_{i j, 0}(s) y^{1-s},
\end{aligned}
$$

where $\delta_{i j}=1$ or 0 according to $i=j$ or not, and

$$
\varphi_{i j, 0}(s)=\sum_{c \neq 0} \frac{(\operatorname{sgn} c) \cdot N_{i j}(c)}{|c|^{2 s}}
$$

with $N_{i j}(c)=\#\left\{0 \leqq d<|c|:\left(\begin{array}{ll}* & * \\ c & d\end{array}\right) \in \sigma_{i}^{-1} \Gamma \sigma_{j}\right\}$. We put

$$
\varphi_{i j}(s)=-\sqrt{-1} \sqrt{\pi} \frac{\Gamma(s)}{\Gamma\left(s+\frac{1}{2}\right)} \varphi_{i j, 0}(s),
$$

and $\Phi(s)=\left(\varphi_{i j}(s)\right)$. Then it is easy to see that the Eisenstein matrix $\Phi(s)$ is a skew-symmetric matrix. The Eisenstein series $E_{i}(z, \phi ; s)$ has no constant terms in its Fourier expansion at any irregular cusp of $\Gamma$.

1.3. First we define the compact part of $E_{i}(z, \phi ; s)$ by

$$
E_{i}^{Y}(z, \phi ; s)= \begin{cases}E_{i}(z, \phi ; s) \cdot-a_{i j, 0}\left(\operatorname{Im}\left(\sigma_{j}^{-1} z\right), \phi ; s\right), & \text { if } \operatorname{Im}\left(\sigma_{j}^{-1} z\right)>Y, \\ E_{i}(z, \phi ; s), & \text { otherwise },\end{cases}
$$

where $Y$ denotes a sufficiently large number. Then, the following MaassSelberg relation of our case may be obtained in a way similar to the proof of Theorem 2.3.2. in Kubota [4]:

$$
\frac{1}{2 \pi}\left(E_{i}^{Y}(z, \phi ; s), E_{i}^{Y}\left(z, \phi ; \bar{s}^{\prime}\right)\right)=\frac{\left.Y^{s+s^{\prime}-1}-\varphi_{i j}(s) \overline{\varphi_{i j}\left(\bar{s}^{\prime}\right.}\right) Y^{-s-s^{\prime}+1}}{s+s^{\prime}-1} \quad(i \neq j) .
$$


We also see that the Eisenstein matrix $\Phi(s)$ converges to a unique unitary matrix $\Phi\left(s_{0}\right)$ when $s$ tends to a point $s_{0}=\frac{1}{2}+\sqrt{-1} r_{0}$. Therefore we have

$$
\begin{aligned}
\Phi\left(s_{0}\right) \Phi\left(1-s_{0}\right) & =\Phi\left(s_{0}\right) \Phi\left(\bar{s}_{0}\right)=-\Phi\left(s_{0}\right) \overline{\Phi\left(s_{0}\right)} \\
& =\Phi\left(s_{0}\right)^{t} \Phi\left(s_{0}\right)=I ;
\end{aligned}
$$

and hence each $E_{i}(z, \phi ; s)$ has a meromorphic continuation to the whole $s$-plane, and the column vector $\mathscr{E}(z, \phi ; s)={ }^{t}\left(E_{1}, E_{2}\right)$ satisfies the functional equation

$$
\mathscr{E}(z, \phi ; s)=\Phi(s) \mathscr{E}(z, \phi ; 1-s) .
$$

Since $\Gamma$ is a discontinuous group of finite type, the integral operator defined by $\omega_{\delta}$ is not generally completely continuous on $L^{2}(\Gamma \backslash \tilde{S})$ and the space $L^{2}(\Gamma \backslash \tilde{S})$ has the following spectral decomposition

$$
L^{2}(T \backslash \tilde{S})=L_{0}^{2}(\Gamma \backslash \tilde{S}) \oplus L_{s p}^{2}(\Gamma \backslash \tilde{S}) \oplus L_{\text {cont }}^{2}(\Gamma \backslash \tilde{S}),
$$

where $L_{0}^{2}$ is the space of non-analytic cusp forms, $L_{s p}^{2}$ is the discrete part of the orthogonal complement of $L_{0}^{2}$ and $L_{\text {cont }}^{2}$ is continuous part of the spectra. By using the meromorphic continuation of the Eisenstein series $E_{i}(z, \phi ; s)$ defined by (2), we put

$$
\tilde{H}_{\delta}\left(z, \phi ; z^{\prime}, \phi^{\prime}\right)=\frac{1}{8 \pi^{2}} \sum_{i=1}^{2} \int_{-\infty}^{\infty} h(r) E_{i}\left(z, \phi ; \frac{1}{2}+\sqrt{-1} r\right) \overline{E_{i}\left(z^{\prime}, \phi^{\prime} ; \frac{1}{2}+\sqrt{-1} r\right)} d r .
$$

Here $h(r)$ denotes the eigenvalue of $\omega_{\delta}$ in $\mathscr{M}(1, \lambda)$ which is given by

$$
h(r)=2^{2+\delta} \pi \frac{\Gamma(1 / 2) \Gamma((1+\delta) / 2)}{\Gamma(\delta) \Gamma(1+\delta / 2)} \Gamma\left(\frac{\delta}{2}+\sqrt{-1} r\right) \Gamma\left(\frac{\delta}{2}-\sqrt{-1} r\right)
$$

with $\lambda=s(s-1)-\frac{5}{4}$ and $s=\frac{1}{2}+\sqrt{-1} r$. We put

$$
K_{\delta}\left(z, \phi ; z^{\prime}, \phi^{\prime}\right)=\sum_{M \in \Gamma} \omega_{\delta}\left(z, \phi ; M\left(z^{\prime}, \phi^{\prime}\right)\right),
$$

and

$$
\tilde{K}_{\delta}=K_{\delta}-\tilde{H}_{\delta}
$$

The integral operator $\tilde{K}_{\delta}$ is uniformly bounded at a neighborhood of each irregular cusp of $\Gamma$. Therefore we may assume that $\kappa_{1}, \kappa_{2}$ is a maximal set of cusps of $\Gamma$. Then the integral operator $\tilde{K}_{\delta}$ is complete continuous on $L^{2}(\Gamma \backslash \tilde{S})$ and has all discrete spectra of $K_{\delta}$. Furthermore, an eigenvalue of $f(z, \phi)$ in $L_{0}^{2}(\Gamma \backslash \tilde{S}) \oplus L_{s p}^{2}(\Gamma \backslash \tilde{S})$ for $\tilde{K}_{\delta}$ is equal to that for $K_{\delta}$ and the 
image of $\tilde{K}_{\delta}$ on it is contained in $L_{0}^{2}(T \backslash \tilde{S})$. Considering the trace of $\tilde{K}_{\delta}$ on $L_{0}^{2}(T \backslash \tilde{S}$ ), we now obtain the following modified trace formula (Selberg [5]):

$$
\begin{aligned}
\sum_{n=1}^{\infty} h\left(\lambda^{(n)}\right) & =\int_{\tilde{D}} \tilde{K}_{\delta}(z, \phi ; z, \phi) d(z, \phi) \\
& =\int_{\tilde{D}}\left\{\sum_{M \in \Gamma} \omega_{\delta}(z, \phi ; M(z, \phi))-\tilde{H}_{\delta}(z, \phi ; z, \phi)\right\} d(z, \phi),
\end{aligned}
$$

where each of $\lambda^{(n)}$ denotes an eigenvalue corresponding to an orthogonal basis $\left\{f^{(n)}\right\}$ for $L_{0}^{2}(T \backslash \tilde{S})$.

\section{§2. A formula for the dimension $d_{1}$}

2.1. We put

$$
\begin{gathered}
\int_{\tilde{D}}\left\{\sum_{M \in \Gamma} \omega_{\delta}(z, \phi ; M(z, \phi))-\tilde{H}_{\delta}(z, \phi ; z, \phi)\right\} d(z, \phi) \\
=J(I)+J(P)+J(R)+J(\infty),
\end{gathered}
$$

where $J(I), J(P), J(R)$, and $J(\infty)$ denote respectively the identity component, the hyperbolic component, the elliptic component, and the parabolic component of the traces. Then the components $J(I), J(P)$ and $J(R)$ were obtained already in [3] and in the following we shall calculate the component $J(\infty)$. Let $\tilde{D}_{i}$ be a fundamental domain of the stabilizer $\Gamma_{i}$ of cusp $\kappa_{i}$ in $\Gamma$ and denote by $\sigma_{i}$ a linear transformation such that $\sigma_{i}^{-1} \Gamma_{i} \sigma_{i}$ $=\Gamma_{0}$. Then we have

$$
J(\infty)=\lim _{Y \rightarrow \infty}\left\{\sum_{i=1}^{2} \int_{\tilde{D}^{Y} \frac{Y}{\substack{M \in \Gamma_{i} \\ M \neq 1}}} \omega_{\delta}(z, \phi ; M(z, \phi)) d(z, \phi)-\int_{\tilde{D}_{Y}} \tilde{H}_{\delta}(z, \phi ; z, \phi) d(z, \phi)\right\},
$$

where $\tilde{D}_{i}^{Y}$ denotes the domain consisting of all points $(z, \phi)$ in $\tilde{D}_{i}$ such that $\operatorname{Im}\left(\sigma_{i}^{-1} z\right)<Y$, and $\tilde{D}_{Y}$ the domain consisting of all $(z, \phi) \in \tilde{D}=\Gamma \backslash \tilde{S}$ such that $\operatorname{Im}\left(\sigma_{i}^{-1} z\right)<Y$ for $i=1,2$.

Making use of a summation formula due to Euler-MacLaurin, we have for the first half of $J(\infty)$ (c.f. [2]),

$$
\int_{\tilde{D}_{i}^{Y}} \sum_{\substack{M \in \Gamma_{i} \\ M \neq 1}} \omega_{\delta}(z, \phi ; M(z, \phi)) d(z, \phi)=2^{2} \pi \frac{\Gamma(1 / 2) I^{\prime}((\delta+1) / 2)}{\Gamma(1+\delta / 2)} \log Y+\alpha(\delta)+o(1)
$$

as $Y \rightarrow \infty$, where $\alpha(\delta)$ denotes a function of $\delta$ such that $\lim _{\delta \rightarrow 0} \delta \alpha(\delta)=0$. For the second half of $J(\infty)$, we have the following by the Maass-Selberg relation (3): 


$$
\begin{aligned}
& \frac{1}{8 \pi^{2}} \int_{\tilde{D}_{Y}} \int_{-\infty}^{\infty} h(r) E_{i}\left(z, \phi ; \frac{1}{2}+\sqrt{-1} r\right) \overline{E_{i}\left(z, \phi ; \frac{1}{2}+\sqrt{-1} r\right)} d r d(z, \phi) \\
& =\frac{1}{8 \pi^{2}} \lim _{t \rightarrow 1 / 2} \int_{\tilde{D}} \int_{-\infty}^{\infty} h(r) E_{i}^{Y}(z, \phi ; t+\sqrt{-1} r) \overline{E_{\imath}^{Y}(z, \phi ; t+\sqrt{-1} r)} d r d(z, \phi)+o(1) \\
& \text { (as } Y \rightarrow \infty \text { ) } \\
& =\frac{1}{4 \pi} \lim _{t \rightarrow 1 / 2} \int_{-\infty}^{\infty} h(r) \frac{Y^{2 t-1}-\varphi_{i j}(s) \overline{\varphi_{i j}(s)} Y^{1-2 t}}{2 t-1} d r+o(1) \quad(\text { as } Y \longrightarrow \infty) \\
& =2^{2} \pi \frac{\Gamma(1 / 2) \Gamma((\delta+1) / 2)}{\Gamma(1+\delta / 2)} \log Y \\
& -\frac{1}{4 \pi} \int_{-\infty}^{\infty} h(r) \varphi_{i j}^{\prime}\left(\frac{1}{2}+\sqrt{-1} r\right) \overline{\varphi_{i j}\left(\frac{1}{2}+\sqrt{-1} r\right)} d r+o(1)
\end{aligned}
$$

as $Y \rightarrow \infty$ and $t \rightarrow \frac{1}{2}$, where $j \neq i$.

2.2. In the following we shall calculate the limit $\lim _{\delta \rightarrow 0} \delta J(\infty)$. By the expression (4) of $h(r)$, we have

$$
h(r) \sim \frac{c(\delta)|\boldsymbol{r}|^{\delta}}{|\boldsymbol{r}| e^{\pi|r|}}
$$

as $r \rightarrow \infty$, where $c(\delta)$ is independent of $r$.

On the other hand, we have $\varphi_{i j}\left(\frac{1}{2}+\sqrt{-1} r\right) \varphi_{i j}\left(\frac{1}{2}-\sqrt{-1} r\right)=-1$ by (3). Therefore

$$
\frac{\varphi_{i j}^{\prime}\left(\frac{1}{2}+\sqrt{-1} r\right)}{\varphi_{i j}\left(\frac{1}{2}+\sqrt{-1} r\right)}=\frac{\varphi_{i j}^{\prime}\left(\frac{1}{2}-\sqrt{-1} r\right)}{\varphi_{i j}\left(\frac{1}{2}-\sqrt{-1} r\right)}
$$

and hence

$$
\int_{-\infty}^{\infty} h(r) \varphi_{i j}^{\prime}\left(\frac{1}{2}+\sqrt{-1} r\right) \overline{\varphi_{i j}\left(\frac{1}{2}+\sqrt{-1} r\right)} d r=\int_{-\infty}^{\infty} h(r) \frac{\varphi_{i j}^{\prime}}{\varphi_{i j}}\left(\frac{1}{2}+\sqrt{-1} r\right) d r .
$$

Now, since the operator $\tilde{K}_{\delta}$ is complete continuous on $L^{2}(\Gamma \backslash \tilde{S})$, we have

$$
\lim _{\delta \rightarrow+0} \delta \int_{-\infty}^{\infty} h(r) \frac{\varphi_{i j}^{\prime}}{\varphi_{i j}}\left(\frac{1}{2}+\sqrt{-1} r\right) d r=0 .
$$

It is clear that the above result, combined with the formulas (*) and (1) obtained in [3] and [2] respectively, proves the following

$$
d_{1}=\frac{1}{2} \operatorname{Res}_{s=0} \zeta^{*}(s) .
$$

Now our main result can be stated as follows.

THEOREM 2. Let $\Gamma$ be a fuchsian group of the first kind not containing 
the element $\left(\begin{array}{rr}-1 & 0 \\ 0 & -1\end{array}\right)$ and suppose that the number of regular cusps of $\Gamma$ is two. Let $d_{1}$ be the dimension for the space consisting of cusp forms of weight 1 with respect to $\Gamma$. Then $d_{1}$ is given by

$$
d_{1}=\frac{1}{2} \operatorname{Res}_{s=0} \zeta^{*}(s),
$$

where $\zeta^{*}(s)$ denotes the Selberg type zeta-function defined in the first part of this paper.

Remark. Let $\Gamma$ be a general discontinuous group of finite type not containing the element $\left(\begin{array}{rr}-1 & 0 \\ 0 & -1\end{array}\right)$. Then, using the properties of the Eisenstein series defined at each regular cusp of $\Gamma$, we can prove that the number of regular cusps of $\Gamma$ is even. We can also prove that in the same way as in the above case, the contribution from parabolic classes to $d_{1}$ vanishes.

\section{REFERENCES}

[1] T. Hiramatsu, On some dimension formula for automorphic forms of weight one I, Nagoya Math. J., 85 (1982), 213-221.

[2] - On some dimension formula for automorphic forms of weight one II, Nagoya Math. J., 105 (1987), 169-186.

[ 3 ] — A formula for the dimension of spaces of cusp forms of weight 1, to appear in Advanced Studies in Pure Math., 15.

[4] T. Kubota, Elementary theory of Eisenstein series, Tokyo-New York: Kodansha and Halsted 1973.

[5] A Selberg, Discontinuous groups and harmonic analysis, in Proc. Int. Math. Congr., Stockholm, 177-189, 1962.

Department of Mathematics

Faculty of Science

Kobe University

Rokko, 657 Japan 